\title{
PREVENTIVE ACTION OF PROXAZOLE IN EXPERIMEN- TALLY INDUCED RENAL HYPERTENSION IN RATS
}

\author{
Silvana BURBERI and Bruno SILVESTRINI \\ Department of Pharmacology, Rescarch Laboratories, A.C.R. Angelini, K, Rome, Italy
}

Received for publication Oetober 20, 1970

Proxazole or 3- $\alpha$-phenylpropyl-5- $\beta$-diethylaminoethyl-1, 2, 4-oxadiazolc cilrate, is an unusual drug because it possesses both anti-inflammatory and antispasmodic properties (1) and because each of these two properties has some uncommon features. The antiinflammatory action takes place mostly against edematous responses and is devoid of ulcerogenic effects which are instead produced by most anti-inflammatory drugs; moreover proxazole prevents indomethacin-induced ulcers without exerting any anti-secretory effect (2-3). The antispasmodic activity results in a specific inhibition of smooth muscle spasm, both at the vascular and at the intestinal level, without significant interferences with the physiologic activity of that tissue (1-4).

The above data suggested a potential interest in the use of proxazole in pathological situations sustained by ischemic-inflammatory processes. Therefore experiments werc undertaken to test proxazole in rats operated according to Grollman (6). In such animals the induced hypertension may be measured and used as an index of renal alterations.

\section{METIODS}

\section{Animals}

Long-Evans rats of both sexes weighing between 170 and $190 \mathrm{~g}$ were used. As no dilference was recorded between male and female rats, the results were grouped together. Each expcriment was performed in groups of animals having homogeneous weight and arterial pressure values. Water and food were administered ad libitum.

Measurement of systolic arterial pressure

Systolic arterial pressure was measured in unanesthetized rats according to the Stanton and White (6) method, using an impulse transducer attached to the tail and registering the pulse beats on a Grass polygraph, mod. 5.

Production of renal hypertension

The Grollman (5) method was employed. Immediately after recording arterial pressure, the rats were anesthetized with ether. The left kidney was then exposed and a figure of 8 ligation was performed around it. Fiften days later the arterial pressure was recorded once again and the contralateral kidney was removed immediately afterwards. The day of nephrectomy was conventionally indicated as 0 time as it corresponds in the majority of the experiments to the beginning of the treatment. 
Drugs

Proxazole citrale, papaverine hydrochloride, reserpine and dihydrochlorothiazide were employed. The doses indicated for the first 2 drugs refer to the salts used. The drugs were administered by mixing them in the diet. The medicated diet was prepared weekly. The average food intake was about $10 \%$ of body weight.

Statistical analysis of results

The significance of results was evaluated using the Student's $t$ or the (chi) methods.

\section{RESULTS}

Trend of renal hypertension

Fig. 1 illustrates the trend of arterial hypertension produced in rats by the Grollman (5) procedure.

No change was observed during the period of time, which is indicated as $-15-0$ days, elapsing between ligature of one kidncy and contralateral nephrectomy. Hypertension was already significant 15 days following the second operation and it showed a rather stable level, ranging between $150-160 \mathrm{~mm}$ of $\mathrm{Hg}$, during the subscquent readings. In control animals no significant variations of arterial pressure were observed.

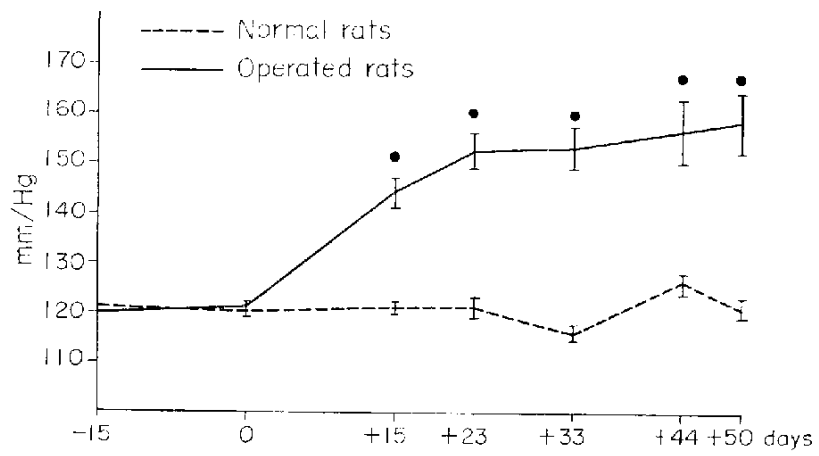

FIG. 1. Trend of arterial pressure in normal rats and in rats operated according to the Grollman (5) method.

In the operated group the time -15 indicates the day of the ligation of one kidney and the time 0 indicates the removal of the opposite kidney. Arterial pressure was recorded immediately before the two operations. 39 normal rats and 117 rats operated according to the Grollman (5) method wore used. Mean values and standard errors are reported.

- $\mathrm{P}<0.001$

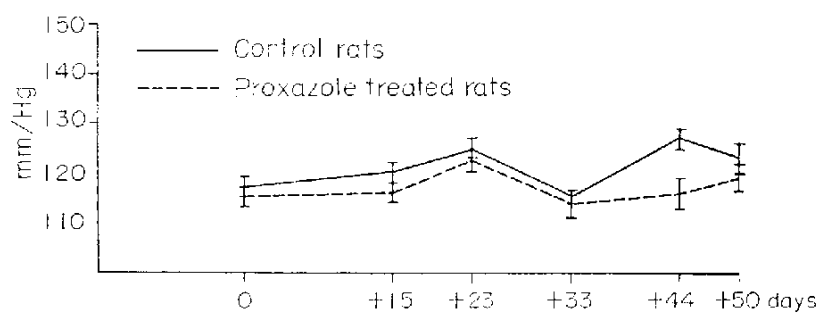

Fic. 2. Effects of proxazole on arterial pressure of normal rats. Proxazole $\langle 0.1 \%$ in the diet $)$ was given throughoul the 50 days experiment. Each group consisted of 20 animals. Mean values and standard errors are reported. 
Effects of proxazole on arterial pressure

Fig. 2 summarizes the results obtained administering proxazole, at an $0.1 \%$ concentration in the diel, to normal rats.

No significant differences were recorded between the arterial pressure of the control group and that of the group treated with proxazole.

Fig. 3 summarizes the results obtained administering proxazole to rats operated according to the Grollman (5) method. The drug was mixed in the diet at an $0.1 \%$ concentration, and it was started from 0 day, corresponding to the day in which nephrectomy took place.

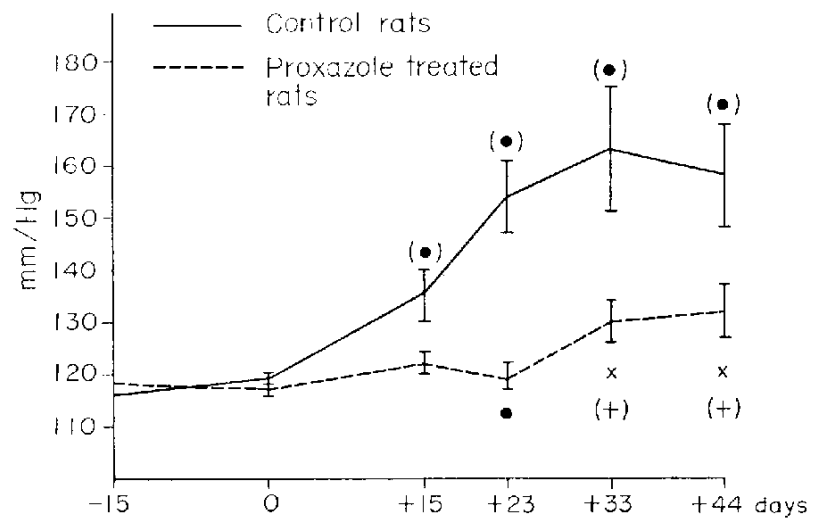

Fig. 3. Effects of proxazole on arterial pressure of rats operated according to Grollman (5).

Proxazole $(0.1 \%$ in the diet $)$ was given for 44 days following nephrectomy. 43 control animals and 29 animals treated with proxazole were used.

Mean values and standard errors are reported.

$x \mathrm{P}<0.05 ;+\mathrm{P}<0.01 ; \mathrm{PP}<0.001$.

The symbols in brackets refer to the comparison with the starting valuc; the others to the comparison between the control group and the treated group.

Control animals developed a marked and statistically significant hypertensive state starting from 15 days after nephrectomy. Blood pressure of animals treated with proxazole remained significantly lower than in control animals all through the experiment; however a slight hypertension, which was statistically significant as compared to initial values, was observed 33 and 44 days after nephrectomy.

In a subsequent experiment rats operated according to the Grollman (5) method were subdivided into two groups: in the first group treatment with proxazole was effected for 50 days after nephrectomy and in the second group from the 50th to the 60th day after nephrectomy. The concentration of proxazole in the diet was $0.1 \%$ and the observation period after nephrectomy was 60 dyas. The results obtained are given in Fig. 4.

In the first group arterial pressure was statistically lower than in the second group, both during treatment and after. By comparing pressure values recorded at various periods with the initial pressure values, it was found that a significant increase in pressure occurred only on the 44th day after nephrectomy. In tch second group proxazole, which 


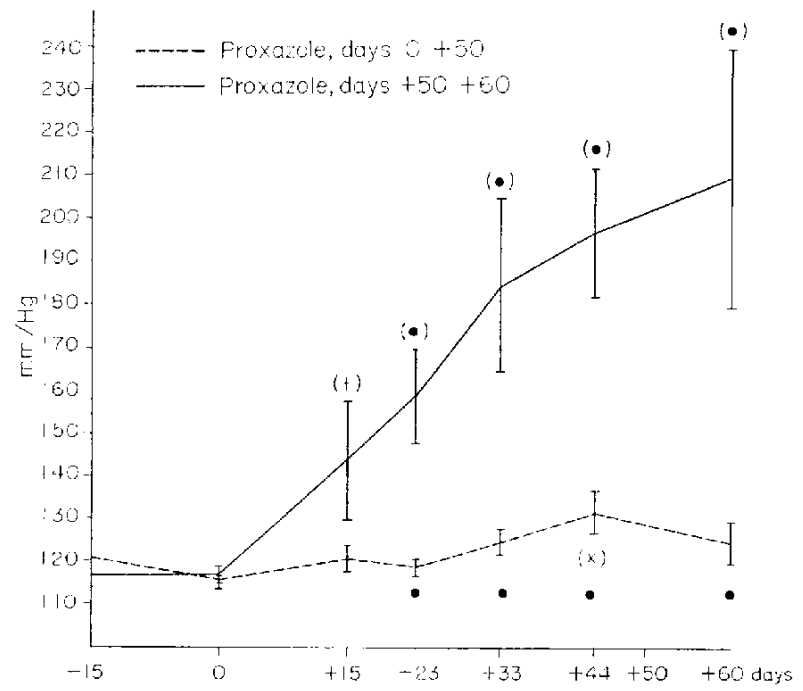

FIG. 4. Effects of proxazole, administered according to different treatment schemes, on the arterial pressure of rats operated according to Grollman (5).

Proxazole $(0.1 \%$ in the diet) was given for 50 days following nephrectomy, in one group, for 10 days starting 50 days after nephrectomy in the second group. The first group consisted of 20 rats, the second of 19 rats.

Mean valucs and standard errors are reported.

$x \quad P<0.05 ;+P<0.01 ; O P<0.001$.

The symbols in brackets refer to the comparison with the starting value; the others to the comparison between the two groups.

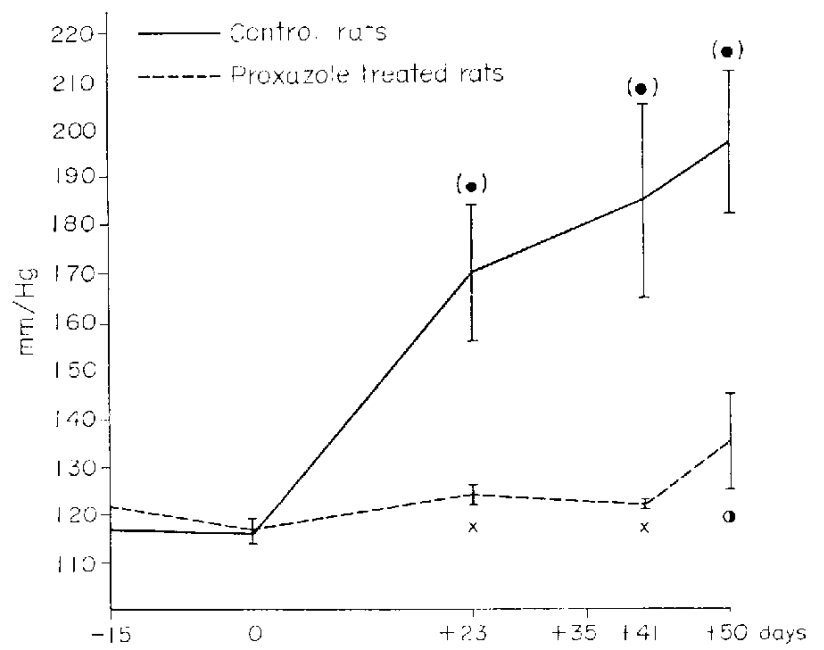

Fic. 5. Effects of proxazole $(0.1 \%$ in the dict), administered for 35 days following nephrectomy, on the arterial pressure of rats operated according to Grollman (5).

Both groups were made up of 10 rats.

Mean values and standard errors were reporled.

$\mathrm{x} P<0.05 ; \quad \mathrm{P}<0.02 ; \mathrm{P}<<0.001$. 
was given from the 50th up to 60th day after nephrectomy, produced no hypotensive effect.

In an altempt to determine the minimum duration of treatment with proxazole required to inhibit the hypertensive process, a group of 15 animals was treated with the drug for 15 days following nephrectomy. Treatment reduced the hypertensive response in comparison to what was observed in the corresponding control group (25 rats), but the effect was not statistically significant. In Fig. 5 are shown the results obtained by treating the animals with proxazole for 35 days following nephrectomy.

In the group treated with proxazole, arterial pressure remained significantly lower than in the control animals, both during treatment and during the 15 days which followed it. Doseleffect relationship with proxazole

A group of animals was treated with a diet containing $0.05 \%$ of the drug. The experiment was performed on 36 rats; 18 of them were treated for 44 days after nephrectomy, while the remaining 18 were used as control animals. Proxazole slightly reduced the hypertensive response, but the difference between the control group did not reach statistical significance. It can therefore be concluded that the effective dose of proxazole ranges between 0.05 and $0.1 \%$ in the diet.

Effects of proxazole on the death rate and body growth of rats operated according to the

Grolliman (5) method

Fig. 6 shows the death rate observed in rats operated according to the Grollman (5) method and then treated with proxazole $(0.1 \%$ in the dict) during the 44 day period following nephrectomy.

The death rate of rats treated with proxazole is much lower than that of the control rats, but the difference is close to the limits of statistical significance $(t-3.151)$ only at the end of the experiment.

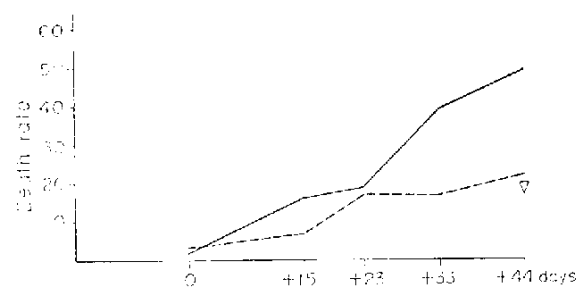

FiG. 6. Effects of proxazole on the death rate of rats operated according to the Grollman (5) method.

Proxazole $(0.1 \%$ in the diet $)$ was given during the 44 days following nephrectomy.

The control group consisted of 43 rats. The group treated with proxazole consisted of 29 rats.

$\nabla: P=0.1-0.05$

The results oblained studying body weight increase are shown in Fig. 7 .

The experiment was conducted on 10 rats treated with proxazole $(0.1 \%$ in the diet) for 60 days following nephrectomy and on 15 control rats. The increase in body weight is greater in the treated rats than in the control ones, but the difference is statistically significant only at the last reading. 


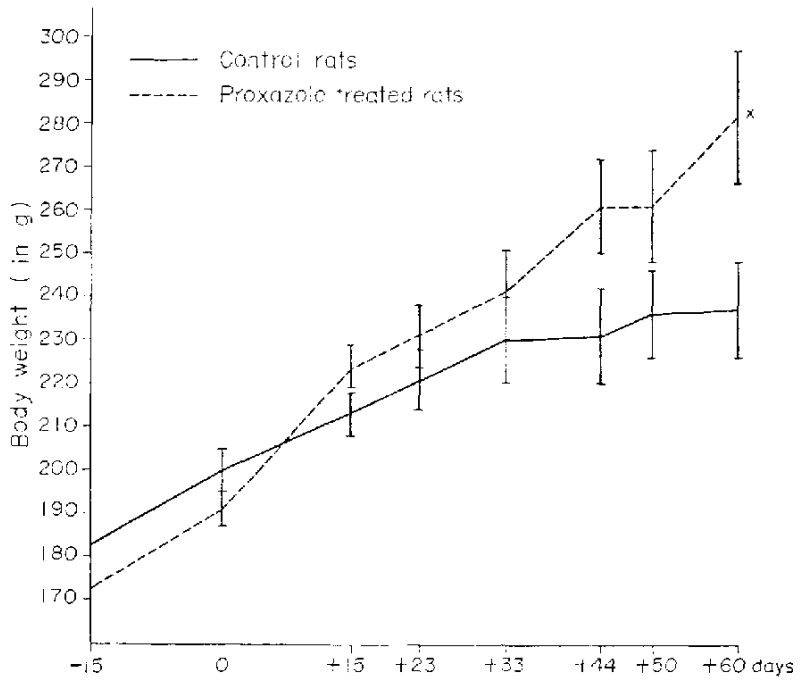

FIG. 7. Effects of proxazole on body weight increase of rats operated according to Grollman (5).

Proxazole $(0.1 \%$ in the diet) was given for 60 days following nephrectomy.

The control group consisted of 15 rats. The group trated with proxazole consisted .. of 10 "rats.

$\mathrm{x}: \mathrm{P}<0.05$.

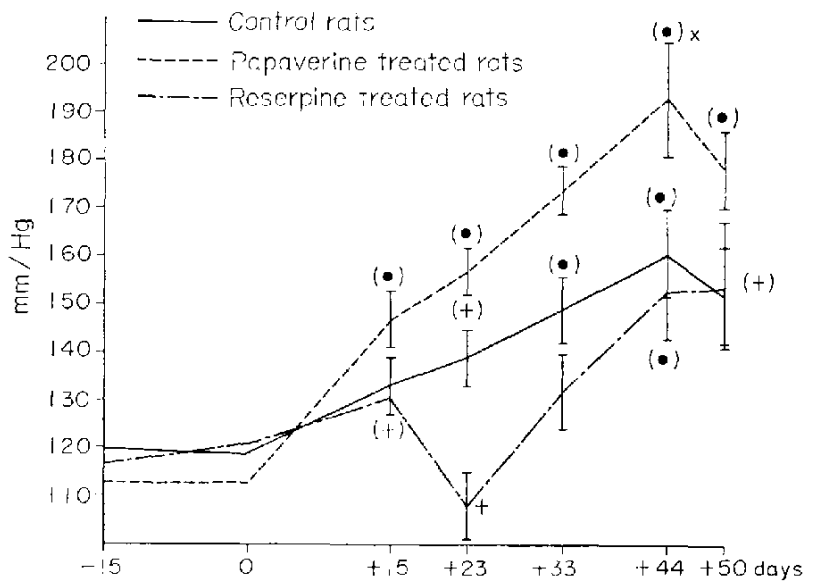

FIG. 8. Effects of papaverine and reserpine on arterial pressure of rats operated according to Grollman (5).

Papaverine $(0.1 \%$ in the diet $)$ and reserpine $(0.0001 \%$ in the diet) were administered for 50 days following nephrectomy. 15 animals were used as controls and 10 animals were used for each experimental trealment.

Mean values and standard errors are reported.

$x \mathrm{P}<0.05 ;+\mathrm{P}<0.01 ; \bigcirc \mathrm{P}<0.001$.

The symbols in brackets refer to the comparison with the starting value; the others to the comparison between each group treated and the control group. 
Effects of papaverine, dihydrochlorothiazide and reserpine on arterial pressure of rats operated according to the Grollman (5) method

Papaverine $(0.1 \%$ in the diet) and reserpine $(0.0001 \%$ in the diet) were given for 50 days starting from nephrectomy.

Results are reported in Fig. 8.

In the control animals the hypertensive response developed rather slowly and statistically significant values, differently from whose observed in previous experiment, were obtained only 23 days after nephrectomy. In the group treated with papaverine the hypertensive response was more marked that in the control animals. In the group treated with rescrpine pressure values were maintained for more than half of the treatment period at lower levels than in the control animals. At the end of the test, however, arterial pressure was the same both in the control group and in the one treated with reserpine.

The test with dihydrochlorothiazide was carried out on 14 rats on the whole, 7 of which were treated with the drug $(0.01 \%$ in the dict for 44 days following nephrectomy) while the other 7 were used as control animals. In both groups a similar hypertensive response was observed.

\section{DISCUSSION}

These experiments demonstrate that the administration of proxazole with the diet to rats operated according to the Grolman (5) method prevents the appearence of hypertension or markedly reduces it. This effect was obtained by starting treatment immediately after nephrectomy and it continued even when treatment was discontinued. It was also observed that proxazole did not exert any hypotensive effect either in normal rats or in rats nephrectomized 50 days beforehand, in which the hypertensive process was already established.

Reserpine, given with the diet to rats operated according to Grollman (5), has produced a hypotensive effect during the first stage of treatment. This result is in accordance with previous tests performed by administering the drug either in a single dose or every day for 4 weeks (7-9). The fact that at the end of the experiment no significant difference was found between control and reserpine treated rats, would suggest that a process of resistance to the drug develops when administration is prolonged. In the same experimental conditions, both papaverine and dihydrochlorothiazide proved to be inactive. Negative results obtained with dihydrochlorothiazide confirm previous investigations (10-11), according to which benzothiadiazine diuretics reduce the desoxycorticosterone-induced hypertension, but not renal hypertension.

The most striking results of these experiments lies in the observation that proxazole possesses a specific preventive action on renal hypertension. Therefore this result shall be discussed in details. In this connection, it must be borne in mind that in the renal hypertension three components can be distinguished:

a) An inflammatory and ischemic process of the kidney.

A protective action at this level must theoretically result in a prevention of the hy- 
pertensive response, without any interference with the arterial pressure of normal animals or of animals in which the hypertension is already established and is linked to degenerative and necrotic phenomena which are irreversible in their nature. Proxazole is, to our knowledge, the first drug showing such a spectrum of effects.

b) The activation of the renin-angiotensin system.

An inhibition, of this system should produce an anti-hypertensive but not hypotensive, effect; namely arterial pressure should be reduced in hypertensive, but not in normal animals. This had been observed by Sen et al. (12) with a phospholipid having anti-renin propertics.

c) The response of structures, which normally control arterial pressure, to renin and angiotensin.

An inhibition of these struetures should produce an hypotensive effect both in normal and hypertensive animals, which is the case of most drugs actually used in the therapy of arterial hypertension.

Therefore the specific preventive action of proxazole on renal hypertension produced in rats according to the Grollman (5) method confirms the working hypothesis that this drug may improve, due to its unusual features, the trophism of tissues suffering from ischemic-inflammatory conditions. This interpretation is in good agreement with experimental observations, according to which proxazole prevents enzymatic depletion produced in the kidney by acute ischemia (13), as well as with clinical results showing a therapeutic action of this drug in kidney discases due to ischemic-inflammatory processes (14).

\section{SUMMARY}

1. The effect of prolonged administration of proxazole on arterial pressure both in normal rats and in rats operated according to the Grollman (5) method were studied. The drug was administered in the diet, starting in most cases from the day of contralateral nephrectomy.

2. Proxazole did not exert any effect either in normal rats or in rats where the hypertensive response had already manifested itsclf and had become stable.

3. When treatment with proxazole began immediately after nephrectomy and was prolonged for at least 35 days, the hypertensive response was greatly reduced and it remaincd at low values even after discontinuing the treatment.

4. Papaverine, reserpine and dihydrochlorothiazide were studied in rats operated according to the Grollman (5) method using the same treatment schedule used with proxazole. Papaverine and dihydrochlorothiazide proved to be devoid of anti-hypertensive effects, whereas reserpine exerted more complex effects: at the heginning of treatment a hypotensive action was observed, but at the end of the experiment pressure values of both treated and control animals were equally high.

5. Results are also discussed from the point of view of their possible therapeutic implications. 


\section{REFERENCES}

1) Sirvthtrini, B. and Poztatti, C.: Arzheimitel Forsch. 13, 798 (1963)

2) Silvestrini, B., Burberi, S., Catanist, B., Cioli, V. and Lisciani, R.: Proccedings of the International Symposium on Proxazole and its Applications in Gastroenterology, Bologna, 10th January Ed. Minerva Medica, Torino (1970)

3) Catanese, B., Lisciani, R. and Silvestrini, B.: Pharmac. Res. Commun. 2, 83 (1970)

4) De Feo, G., Piccinfeld, D. and Silvestrini, B.: Farmaco (Ed. prat.) 26, 123 (1971)

5) Grollman, A.: Proc. Soc. exp. Biol. Med. 57, 102 (1944)

6) Stanton, H.C. And White, J.B.: Archs int. Pharmacodyn. Thér. 154, 351 (1965)

7) Grollman, A.: J. Phamac. exp. Ther. 114, 263 (1955)

8) Smitri, T.II.F. and Rossi, G.V.: J. Pharmac. exp. Ther. 135, 367 (1962)

9) Willard, P. and Beckhelm, G.A.: Aicls int. Pharmacodyn. Thér. 173, 11 (1968)

10) Plummer, A.J. and Yonkman, F.F.: J. chem. Education 37, 179 (1960)

11) Tobian, L. And Colliti, K, : Proc. Soc. exp. Biol. Med, 115, 196 (1964)

12) Sfi, S., Smiry, R.R. and Bumpus, F.M.: Am. J. Physiol. 214, 337 (1968)

13) Esposito, G. And Gull.A, P.: Gazz. int. Mod. Chir. 73, (1968)

14) Mioli, V., Almhrtaz.j, A., Vangiflista, A. and Bonomini, V.: Clin. ter. 50, 437 (1969) 